\title{
Supporting Information for Coherent Vibrational Probes of Hydrogen Bond Structure Following Ultrafast Electron Transfer
}

\author{
Aaron S. Rury*, Shayne Sorenson, and Jahan M. Dawlaty* \\ Department of Chemistry, University of Southern California \\ E-mail: arury@usc.edu
}

*To whom correspondence should be addressed 


\section{Visible Reflection of Monoclinic Quinhydrone}

Figure S1 shows the reflectivity spectrum of a monoclinic quinhydrone single crystal reported previously showing the peak of the charge transfer transition near $2.0 \mathrm{eV} .^{1}$

\section{Ultrafast Transient Reflectivity and Phonon Coherence}

\section{Spectra}

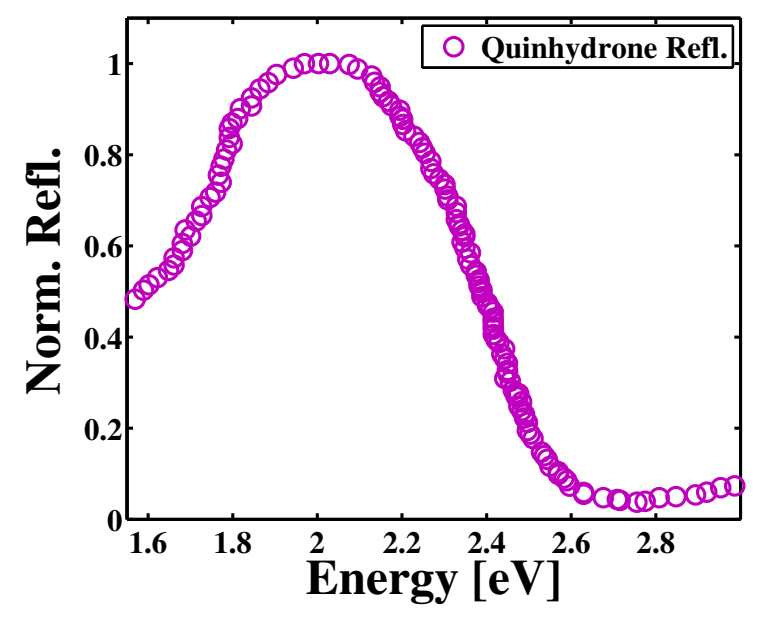

Figure S1: Normalized reflectivity spectrum of a monoclinic quinhydrone single crystal reported by Mitani et al. in Ref. (1) showing peak of charge transfer resonance near $2.0 \mathrm{eV}$.

A schematic showing the general design of the ultrafast spectroscopic measurements highlighted in the manuscript is shown in Figure S2. Despite the broad bandwidth necessary to produce ultrafast pulse of mid-infrared light, the frequency content of the probe pulses from our OPA does not span the entire region of the ground state $\mathrm{OH}$ stretch of quinhydrone. Therefore, to capture the ultrafast dynamics of quinhydrone in this region of interest, we tuned the OPA that generates the probe pulse to three distinct frequencies, as depicted in Figure 2 of the manuscript. In order to create a single, broadband vibrational transient reflectivity (vTR) spectrum, we examined the TR of the probe at each frequency, which typically spans $\sim 300 \mathrm{~cm}^{-1}$, at specific pump-probe delay times to determine their spectral 


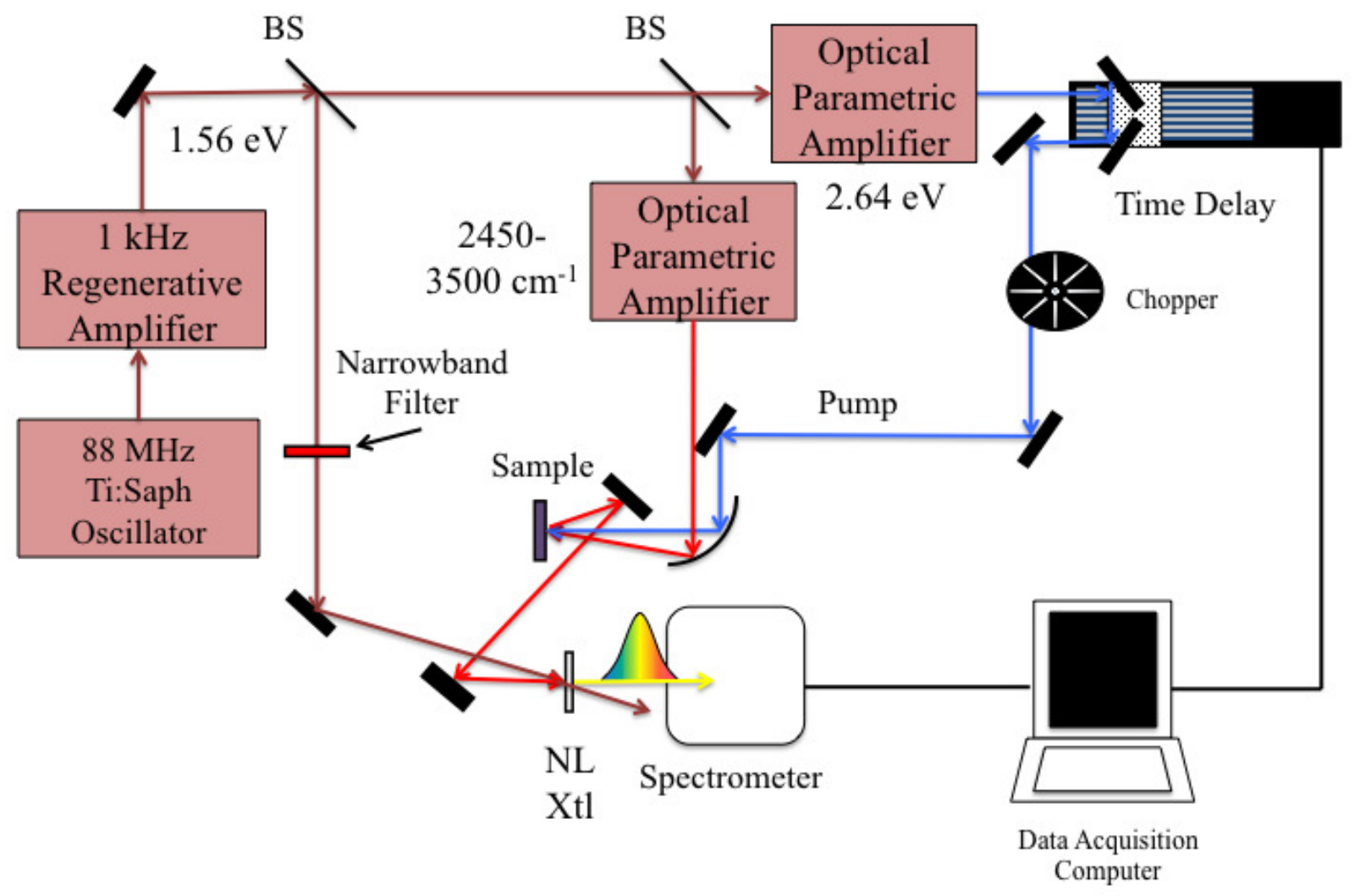

Figure S2: Schematic of the visible pump, mid-infrared ultrafast pump probe experimental design. BS is beam splitter and NL Xtl is a magnesium oxide-doped lithium niobate crystal designed for sum frequency generation of the near-infrared and mid-infrared ultrafast pulses. 


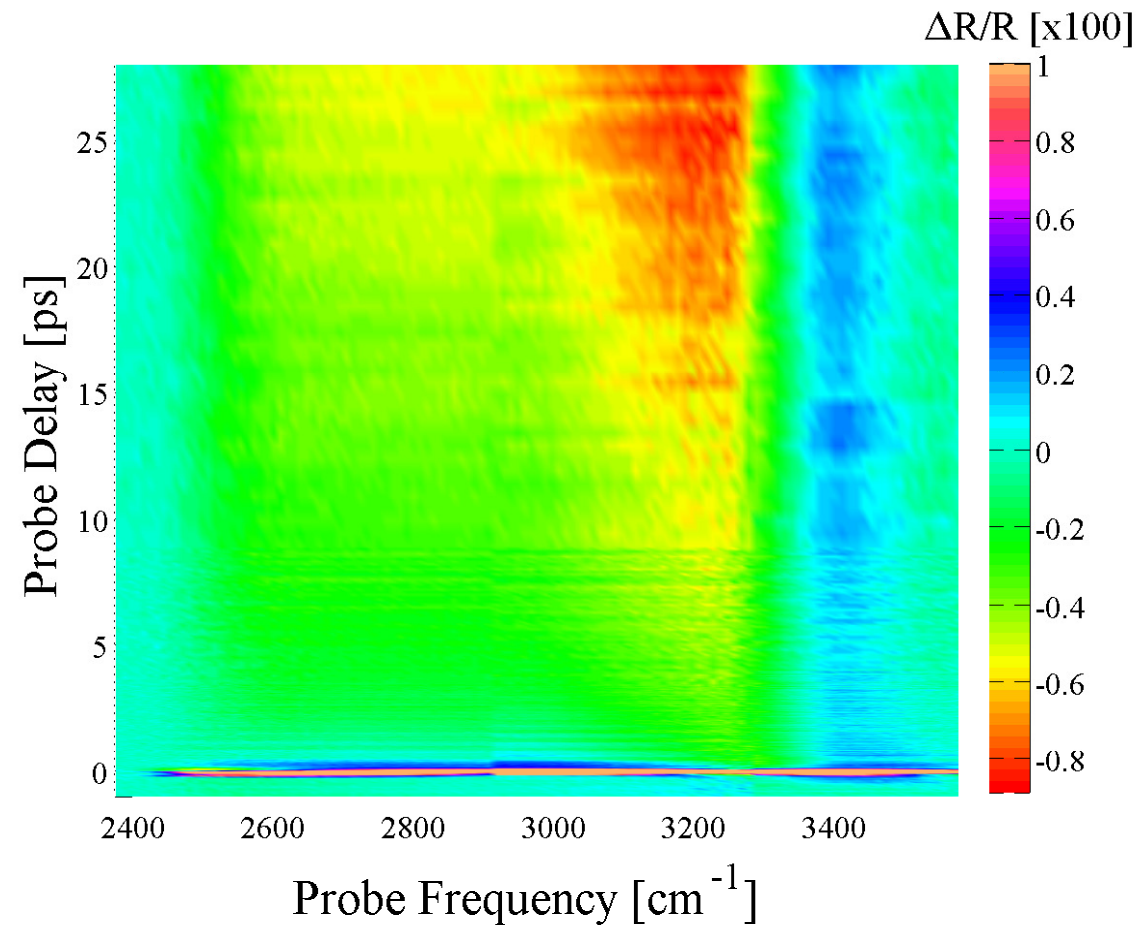

Figure S3: Long-time ultrafast vibrational transient reflectivity spectrum of a monoclinic quinhydrone single crystal excited at $2.64 \mathrm{eV}$ and probed in the region of the ground state $\mathrm{OH}$ stretching vibration. Magnitude of the change in reflectivity signal, $\Delta R / R$, is reported in percent. 
overlap. We then stitched together the different regions to create a single broadband vTR spectrum by scaling the TR spectrum measured for the probe pulse at location 2 in Figure 2 by a factor of 1.5 and no scaling for the other spectra. Figure S2 shows the total vTR spectrum from $2400 \mathrm{~cm}^{-1}$ to $3550 \mathrm{~cm}^{-1}$ excited by a $2.64 \mathrm{eV}$ pump pulse for pump-probe delays between -1 and 28 ps.

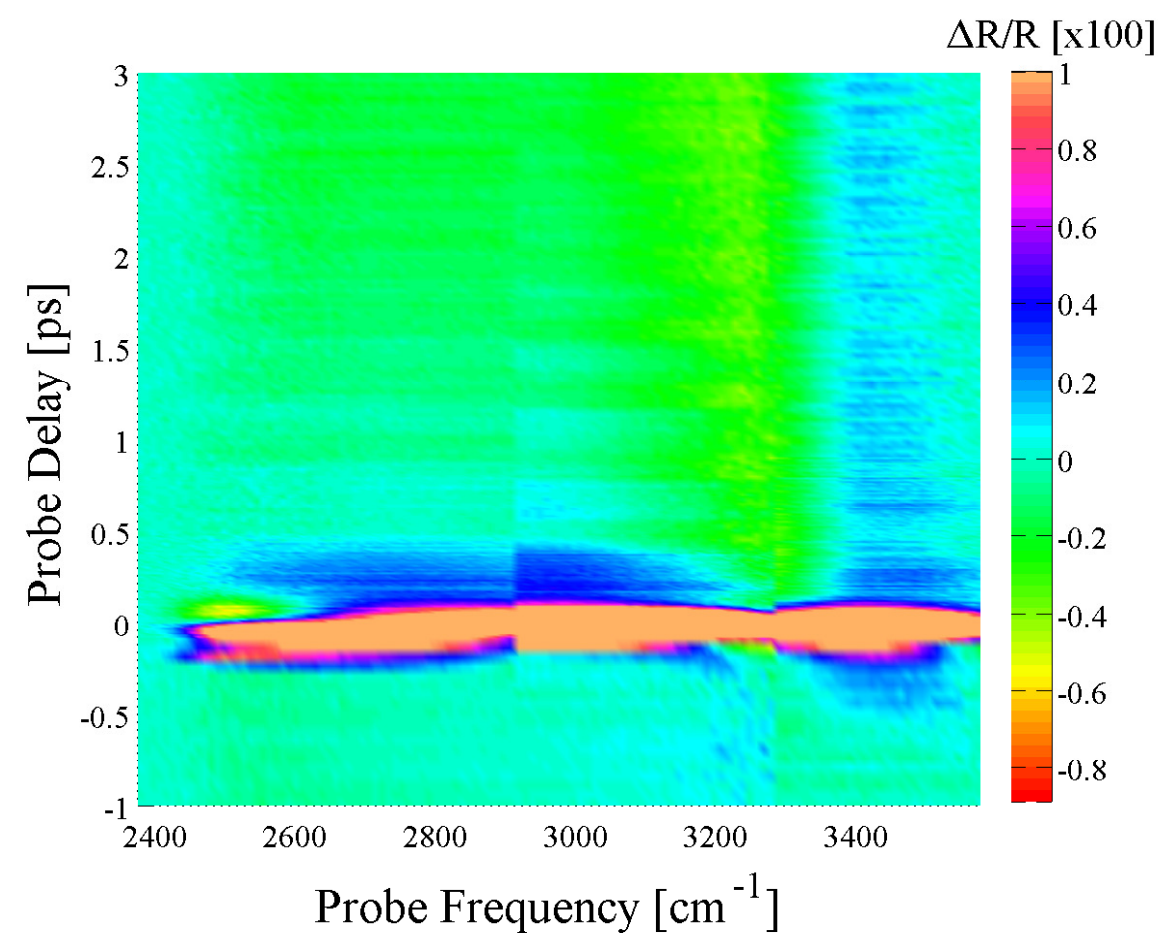

Figure S4: Short-time ultrafast vibrational transient reflectivity spectrum of a monoclinic quinhydrone single crystal excited at $2.64 \mathrm{eV}$ and probed in the region of the ground state $\mathrm{OH}$ stretching vibration. Magnitude of the signal is reported in percent change in reflectivity, $\Delta R / R$. Horizontal stripes in spectrum correspond to quantum beats of lattice phonon vibrational states excited by the ultrafast pump pulse.

Figure S3 shows the full vTR spectrum excited at $2.64 \mathrm{eV}$ of a single monoclinic crystal of quinhydrone for pump-probe delays between -1 and 3 ps. Oscillations appear as horizontal stripes that correspond to the coherent superposition of lattice phonon vibrational states excited by the ultrafast pump pulse. The spectrum of frequencies of these oscillations integrated across the total band of our measurement is shown in Figure S4. Previous studies of the vibrational spectra of quinhydrone provide a baseline for the analysis of the coupling of the $\mathrm{OH}$ stretch with phonons of the crystal's lattice. In particular, as noted in the text 


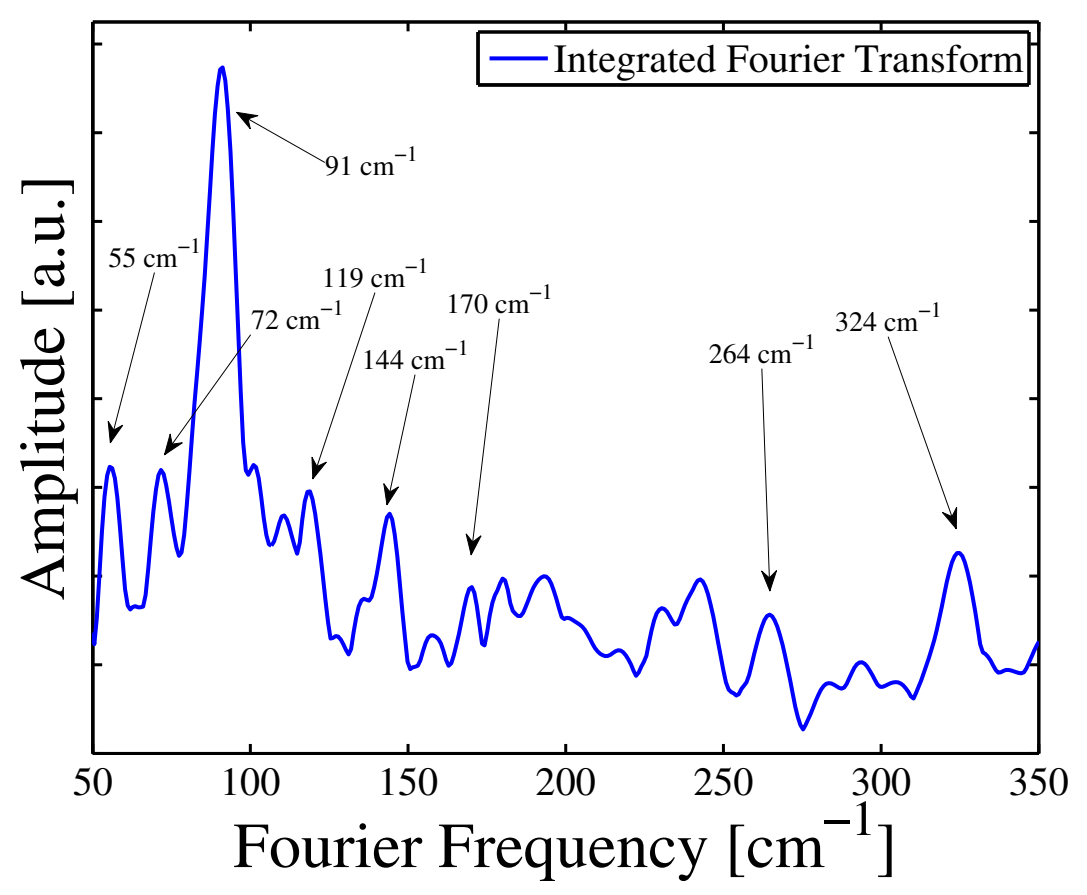

Figure S5: Integrated Fourier transform of the ultrafast vibrational transient reflectivity spectrum of a monoclinic quinhydrone single crystal excited at $2.64 \mathrm{eV}$ and probed in the region of the ground state $\mathrm{OH}$ stretching vibration. Arrows indicate 8 frequencies of interest for which we have examine molecular vibration-phonon coupling spectra. 
of the manuscript, the peaks at $72 \mathrm{~cm}^{-1}$ and $144 \mathrm{~cm}^{-1}$ likely correspond to lattice phonons previously observed in IR absorption measurements. ${ }^{1,2}$ Their appearance in our data must occur via an anharmonic coupling between these lattice phonons and at least one Ramanactive phonon. In addition to these IR-active phonons that appear in the Fourier spectrum, at least three features appear at frequencies close to peaks in the resonance Raman spectra shown in Figures 3 and 5 of the manuscript, at $91 \mathrm{~cm}^{-1}, 170 \mathrm{~cm}^{-1}$, and $264 \mathrm{~cm}^{-1}$. Also, a peak appears in the Fourier spectrum at $55 \mathrm{~cm}^{-1}$ that may correspond to a Raman-active mode not observed in our resonance Raman measurements, but is present in ultrafast transient reflectivity measurements pumped at $1.82 \mathrm{eV}$ and probed between $2.0 \mathrm{eV}$ and $2.6 \mathrm{eV} .^{3}$ However, the assignment of the activity of this feature remains tenuous at best, given our experimental data. We point out the positions of all of these features along with a peak at $324 \mathrm{~cm}^{-1}$ in Figure S4.

To further investigate the coupling of the $\mathrm{OH}$ stretch of quinhydrone to these lattice phonons, we have undertaken a phonon coherence spectroscopic analysis. The singular value decomposition (SVD) analysis presented previously allows calculation of spectra across the full band of our measurement that report both the amplitude and phase of the oscillations in the ultrafast vTR spectrum of Figure S3 as a function of probe frequency. Figure S5 shows these spectra for the 8 features highlighted in Figure S4, which we will call phonon coherence spectra (PCS). We have added dashed vertical lines to each of the spectra in Figure S5 to denote the frequencies at which the TR from each probe pulse have been stitched together using the process describe above. Analysis of the remaining features in the Fourier spectrum of Figure S5 necessitates further motivation from either experiment or theory that neither we nor others have yet completed. Therefore, we do not show the PCS of these features here. To understand how the spectra of Figure S5 report coupling between the $\mathrm{OH}$ and a given phonon mode we must consider one specific attribute of both the amplitude and phase of these spectra.

Several authors have shown the spectral properties of the amplitude and phase of a vibra- 

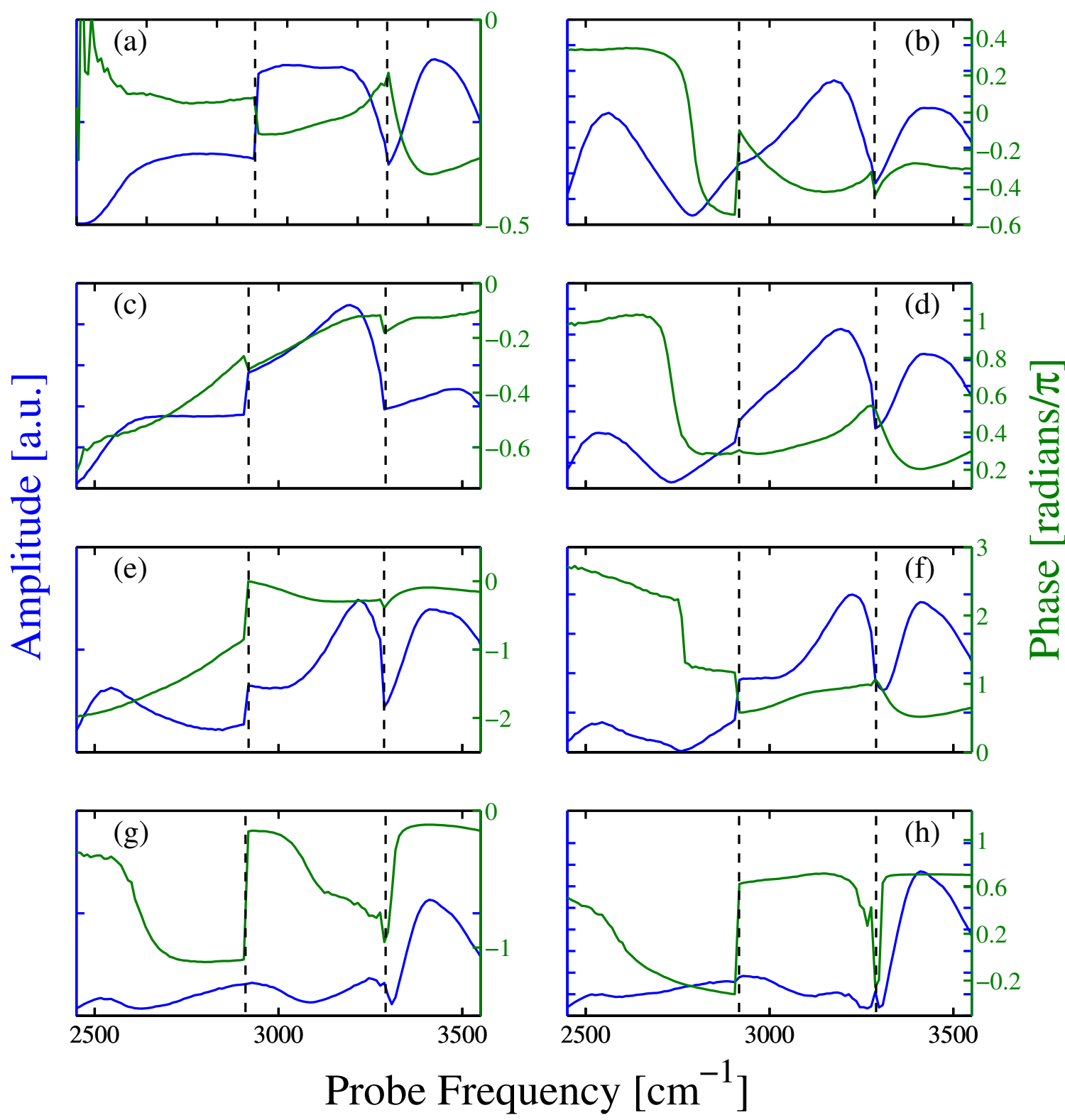

Figure S6: Amplitude (blue) and phase (green) of the phonon coherence spectra corresponding to the peaks at $55 \mathrm{~cm}^{-1}$ (a), $72 \mathrm{~cm}^{-1}$ (b), $91 \mathrm{~cm}^{-1}$ (c), $119 \mathrm{~cm}^{-1}$ (d), $144 \mathrm{~cm}^{-1}$ (e), $170 \mathrm{~cm}^{-1}$ (f), $264 \mathrm{~cm}^{-1}$ (g) and $324 \mathrm{~cm}^{-1}$ (h) in the integrated Fourier spectrum of Figure S6. Dashed vertical denote the positions at which the TR of each probe pulse were stitched together, as explained in text.

tional quantum beat spectrum, such as the PCS, should change when the vibration driving the quantum beats couples to a transition resonant with the probe energy. ${ }^{4-8}$ Specifically, at the peak of this transition one should observe a dip in the amplitude and a shift in the 
phase. Both of these effects stem from the destructive interference of competing coherent Raman, four-wave mixing processes that occurs at the peak of the transition. While Raman scattering processes resonant with electronic transitions like those observed previously should not contribute to our measurements probed in the mid-IR, other Raman processes due to anharmonic coupling can still provide the necessary physical mechanism to use the amplitude and phase of the PCS to identify transitions coupled to specific lattice phonons. ${ }^{9}$ Therefore, we can determine if a given lattice phonon of quinhydrone couples to a transition in the region of the ground state $\mathrm{OH}$ stretching vibration by examining the structure of the PCS.

Inspection of Figure S5 shows that the lattice phonons driving the quantum beats in our ultrafast vTR measurements vary significantly in their coupling to transitions in the spectral region we probe. Specifically, outside of the stitching regions, the PCS corresponding to the features of the Fourier spectrum at $55 \mathrm{~cm}^{-1}, 91 \mathrm{~cm}^{-1}$ and $144 \mathrm{~cm}^{-1}$ in panels (a), (c) and (e), respectively, do not show any distinct dips in their amplitude or shifts in their phase. This fact seems to indicate that these vibrations do not directly couple to mid-infrared transitions present in the charge separated state of quinhydrone in our probe bandwidth. In contrast, the other 5 PCS all show at least one distinct dip in amplitude with a coincident shift in phase. These attributes of the PCS establish the ability of these phonons to modulate the resonant energy of a transition present in the excited state of quinhydrone within the probe bandwidth.

\section{Analytical Modeling}

To understand the information reported by the phonon coherence spectra shown in the manuscript as well as Figure S5, we have undertaken analytical modeling of these spectra based on the work of Kumar et al.. ${ }^{4,5}$ In their work, Kumar et al. use the semiclassical phase space Wigner representation of the density matrix to treat oscillations like those we observe 
in our measurements as wave packet motion on potential energy surfaces associated with the different degrees of freedom of a molecule or material. Following electronic excitation, one can then base the coherent nuclear dynamics of the material after interaction with the pump pulse on the initial position, $\bar{Q}(0)$, and momentum, $\bar{P}(0)$ of the wave packet in the ground and excited electronic states. In this treatment, the frequency dependence of the first moment of the amplitude and phase of a ground state wave packet becomes, ${ }^{5}$

$$
\left|A_{1 g}(\nu)\right| \exp \left[-i \phi_{1 g}(\nu)\right]=\bar{Q}_{g}(0)+i \bar{P}_{g}(0)
$$

while frequency dependence of the first moment of the amplitude and phase of an excited state wave packet becomes

$$
\left|A_{1 e}(\nu)\right| \exp \left[-i \phi_{1 e}(\nu)\right]=\bar{Q}_{e}(0),
$$

since the momentum imparted on the nuclear wavepacket in the excited state by the pump pulse is zero.

However, we must take care in mapping these previous treatments directly onto our measurements. Specifically, unlike the vast majority of experimental studies on the coupling electronic and vibrational degrees of freedom undertaken thus far, we use a non-degenerate, two-color pump-probe experimental design to assess coupling in our measurements. Therefore, appropriate account of this distinction is necessary to model our results. Fortunately, the work of Kumar et al. already considered the case of a three level system in modeling non-radiative transitions among electronic states to explain the ultrafast dynamics of myoglobin. ${ }^{4}$ Therefore, by using a three level model including the contributions of the ground and excited states of the $\mathrm{OH}$ stretch in the charge separated electronically excited state of quinhydrone we can use the results of Kumar et al. to explain the behavior of the PCS we measure. To do so we make the well-separated pulse approximation and allow the pump to make coherent wave packets of the lattice phonon vibrational states in both the ground and 
excited states of $\mathrm{OH}$ stretching vibration upon optical excitation.

Furthermore, in the case of our measurements the pump pulse of $\sim 50$ fs is shorter than the period of the lattice phonon vibrations we consider. Therefore, we can simplify the expressions for the initial position and momentum of the wave packets in the impulsive $(I)$ limit as, ${ }^{5}$

$$
\begin{gathered}
\bar{Q}_{g}^{(I)}(0)=-\frac{\left|\mu_{e g}\right|^{2} E_{0}^{2}(2 \bar{n}+1) \nu_{p h} \Delta}{8 \pi h^{2} N_{g}} \int_{0}^{\infty} d \nu \bar{G}^{2}\left(\nu-\nu_{c}\right) \frac{\partial \Phi_{I}(\nu)}{\partial \nu} \\
\bar{P}_{g}^{(I)}(0)=\frac{\left|\mu_{e g}\right|^{2} E_{0}^{2} \nu_{p h} \Delta}{8 \pi h^{2} N_{g}} \int_{0}^{\infty} d \nu \bar{G}^{2}\left(\nu-\nu_{c}\right) \frac{\partial \Phi_{R}(\nu)}{\partial \nu} \\
\bar{Q}_{e}^{(I)}(0)=-\frac{\left|\mu_{e g}\right|^{2} E_{0}^{2} \Delta}{4 \pi h^{2} N_{e}} \int_{0}^{\infty} d \nu \bar{G}^{2}\left(\nu-\nu_{c}\right)\left[\Phi_{I}(\nu)-\bar{n} \nu_{p h} \frac{\partial \Phi_{I}(\nu)}{\partial \nu}\right]
\end{gathered}
$$

where $\mu_{e g}$ is the transition dipole moment resonant with the probe pulse, $E_{0}$ is the probe electric field, $N_{g}$ and $N_{e}$ are the ground and excited vibrational state populations, $\bar{n}$ is the thermal occupancy of the lattice phonon of frequency $\nu_{p h}$ given by $1 /\left[e^{\left(h \nu_{p h} / k_{B} T\right)}-1\right]$ and $\bar{G}\left(\nu-\nu_{c}\right)$ is the spectral envelope of the probe pulse centered at a frequency $\nu_{c}$, which we take to be Gaussian as described previously. ${ }^{5} \Delta=d \sqrt{\mu_{m} \nu_{p h} /(2 h)}$ is the dimensionless displacement that leads to the wave packet motion being probed where $\mathrm{d}$ is physical displacement of the involved states and $\mu_{m}$ is the reduced mass of the monoclinic quinhydrone unit cell, $\mu_{m}=m_{H Q} m_{B Q} /\left(m_{H Q}+m_{B Q}\right) \cdot{ }^{10} \Phi_{I}(\nu)$ and $\Phi_{R}(\nu)$ are the imaginary and real parts, respectively, of the transition coupled to a given lattice phonon mode, related to one another by the Kramers-Kronig relation. ${ }^{11}$ For the purposes of our analysis, we have presumed that the transitions coupled to the lattice phonons of quinhydrone are inhomogeneously broadened by the lattice mode of interest in the same fashion as proposed by Kumar et al. ${ }^{4}$ Therefore, the transitions are parameterized as, 


$$
\Phi_{I}(\nu)=\sqrt{\frac{\pi}{2 \sigma_{T}(\nu)^{2}}} e^{-\left(\nu-\nu_{0}\right)^{2} / 2 \sigma_{T}(\nu)^{2}},
$$

where

$$
\begin{gathered}
\sigma_{T}(\nu)=\frac{2 \sigma_{T}}{1+e^{\left[a\left(\nu-\nu_{0}\right)\right]}}, \\
\sigma_{T}=\Delta \sqrt{k_{B} T \nu_{p h} / h} .
\end{gathered}
$$

We have also phenomenologically added the possibility for an asymmetric line shape of the transition in Eq. (S5a), as done previously. ${ }^{12}$ For clarity, we also note that we use $h$ instead of $\hbar$, contrasting with the specific notation of Champion and co-workers. ${ }^{4,5}$ Therefore, for a lattice phonon of a given frequency, $\nu_{p h}$, there are four possible inputs that define the transitions we use to model the PCS spectra: a displacement, $d$, in $\AA$, a temperature, $T$, a center frequency $\nu_{0}$ and an asymmetry, $a$. However, the temperature $T$ will either correspond to the temperature at which we perform the experiment or that of an ensemble of phonons thermalized by the energy of the visible pump pulse, as further discussed below.

Of the modes whose PCS spectra show attributes consistent with the modulation of the energy of a transition, we focus on the $72 \mathrm{~cm}^{-1}$ and $170 \mathrm{~cm}^{-1}$ peaks of Figure S6. This focus is partially justified based on the presence of a $172 \mathrm{~cm}^{-1}$ peak in our previous visible pump, visible probe measurements whose electron-phonon coupling spectrum shows that this mode modulates an electronic transition resonant near $\sim 2.0 \mathrm{eV}\left(\sim 16100 \mathrm{~cm}^{-1}\right) .{ }^{8}$ Therefore, any coupling of this lattice phonon to vibrational transitions related to proton dynamics could mean that this mode couples to both electron and proton behavior in the same excited electronic of quinhydrone. In addition, the $72 \mathrm{~cm}^{-1}$ mode lies at a frequency close to that of BQ $\cdots H Q$ mode assigned previously. ${ }^{2}$ Therefore, this mode should sensitively probe hydrogen 
bonding in the charge separated state in quinhydrone.

To qualitatively model the PCS spectra, we utilize a Kramers-Kronig transformation code provided by Lucarini et al. to calculate $\Phi_{R}(\nu)$ from $\Phi_{I}(\nu)$ given the set of inputs described above. ${ }^{11}$ We then calculate the initial position and momentum of the ground and excited states as defined by Eqs. (S3a-S3c). However, since we resonantly probed vibrations in our measurement, the physical meaning of the ground and excited states in our analysis no longer correspond to the electronic degrees of freedom of quinhydrone, as was considered by Kumar et al.. These states now correspond to the excited and ground potential energy surfaces of the $\mathrm{OH}$ along the lattice phonon coordinates, as depicted in Figure 4 of the manuscript. As stated above, we make no presumption concerning the ability of the pump pulse to form coherent lattice phonon superpositions in the ground or excited states of the $\mathrm{OH}$ vibration. Therefore, we have also used linear combinations of the ground and excited state PCS defined in Eqs. (S1) and (S2) to reproduce the experimental spectra. After each calculation we compare the experimental and calculated PCS using Eqs. (S1)-(S5) to check the ability of the model spectrum to reproduce the qualitative features of our experimental spectrum. We repeat this process until we converge on reasonable qualitative agreement between model and experimental spectra.

Comparison of model and experimental PCS associated with the $72 \mathrm{~cm}^{-1}$ and $170 \mathrm{~cm}^{-1}$ peaks of Figure S4 for $T=298 \mathrm{~K}$ in the probe frequency region $2400 \mathrm{~cm}^{-1}$ through 2900 $\mathrm{cm}^{-1}$ are shown in Figures 3 and 4 of the manuscript, respectively. Upon inspection of these Figures, we find that our method provides qualitative agreement between the model and experimental spectra, especially at probe frequencies near the dip of the amplitude and shift of the phase, but there is some deviation at the lowest probe frequencies of our measurements. The probe overlap with atmospheric $\mathrm{CO}_{2}$ likely explains this deviation between the model and experimental spectra. We present the parameters used to calculate the model PCS in Table 1. In the case of both transitions, we find very broad line shapes, albeit with different symmetries. Figure 9 of the manuscript shows that while we find a symmetric transition 
coupled to the $170 \mathrm{~cm}^{-1}$, we need to add an asymmetry to the line shape of the transition coupled to the $72 \mathrm{~cm}^{-1}$ peak. Given the parameters we've used to calculate the model PCS, it seems that these broad line widths originate in the substantial displacement of the excited $\mathrm{OH}$ stretch vibrational state relative to its ground state along these phonon coordinates. However, it is not clear that our experimental conditions justify a temperature of $298 \mathrm{~K}$.

As pointed out in the text of the manuscript, by exciting quinhydrone at $2.64 \mathrm{eV}$ we deposit several 100s of meV of energy in excess of the optically induced electron transfer transition between $\mathrm{HQ}$ and BQ. If quinhydrone dissipates this excess energy into its nuclear degrees of freedom on a time scale over which we analyze the PCS, then we may be incorrectly inferring the source of the broadening of the transitions coupled to its lattice phonons. In order to reproduce the qualitative behavior of the experimental spectra at $298 \mathrm{~K}$, we have adjusted the displacement, $d$. However, a similar effect could also occur by increasing $T$. Eq. (6b) shows that the line width used to produce the transitions depends on temperature as $\sqrt{T}$. Therefore, as $T$ increases, so should the line width of the transitions coupled to the lattice phonons of quinhydrone. However, increasing the temperature also produces other effects that must reproduce our experimental findings to merit considering this possibility further.

Specifically, by increasing the temperature, we change the average occupancy of the lattice phonon vibrational states, $\bar{n}$. For higher temperatures, $\bar{n}$ should increase. Eqs. (S3a), (S3b), and (S3c) show that as $\bar{n}$ changes, so should the initial position of the wave packets in the ground and excited states of the $\mathrm{OH}$ stretch, while no change should occur for the initial momentum. Since the position and momentum relate to one another via a derivative, changes in their respective contribution to Eq. (S2) will result in significant changes to the structure of the ground state phonon coherence spectrum as a function of temperature.

One of the key attributes necessary for the qualitative reproduction of the experimental PCS is its slope away from the minimum of the amplitude's dip toward both lower and higher frequencies. In the case of the experimental spectrum corresponding to the $72 \mathrm{~cm}^{-1}$ 
quantum beat, we find that the slope toward lower frequencies is greater than toward higher frequencies. For the case of the asymmetric transition shown in Figure 6 of the manuscript, we can reproduce these attributes of the structure of the PCS. In contrast, we cannot reproduce the structure of the PCS in panel (b) of Figure S6 in the presence of an ensemble of $72 \mathrm{~cm}^{-1}$ lattice phonons thermalized by the excess energy of the $2.64 \mathrm{eV}$ pump pulse above the charge-transfer optical gap.

\section{References}

(1) Mitani, T.; Saito, G. Cooperative Phenomena Associated with Electron- and ProtonTransfer in Charge-Transfer Crystals. Synthetic Metals 1988, 27, B499-B508.

(2) Fukushima, K.; Sakurada, M. Lattice Vibrations of Quinhydrone and the Intermolecular Potential in the Crystal. The Journal of Physical Chemistry 1976, 80, 1367-1373.

(3) Rury, A. S.; Sorenson, S.; Dawlaty, J. M. Intermolecular Electron Transfer from Intramolecular Excitation and Coherent Acoustic Phonon Generation in a HydrogenBonded Charge-Transfer Solid. The Journal of Chemical Physics 2016, 144, 104701.

(4) Kumar, A. T. N.; Rosca, F.; Widom, A.; Champion, P. M. Investigations of Amplitude and Phase Excitation Profiles in Femtosecond Coherence Spectroscopy. The Journal of Chemical Physics 2001, 114, 701.

(5) Kumar, A. T. N.; Rosca, F.; Widom, A.; Champion, P. M. Investigations of Ultrafast Nuclear Response Induced by Resonant and Nonresonant Laser Pulses. The Journal of Chemical Physics 2001, 114, 6795.

(6) Lüer, L.; Gadermaier, C.; Crochet, J.; Hertel, T.; Brida, D.; Lanzani, G. Coherent Phonon Dynamics in Semiconducting Carbon Nanotubes: A Quantitative Study of Electron-Phonon Coupling. Physical Review Letters 2009, 102, 127401. 
(7) Kobayashi, T.; Nie, Z.; Du, J.; Okamura, K.; Kataura, H.; Sakakibara, Y.; Miyata, Y. Electronic Relaxation and Coherent Phonon Dynamics in Semiconducting Single-Walled Carbon Nanotubes with Several Chiralities. Physical Review B 2013, 88,035424 .

(8) Rury, A. S.; Sorenson, S.; Driscoll, E.; Dawlaty, J. M. Electronic State-Resolved Electron-Phonon Coupling in an Organic Charge Transfer Material from Broadband Quantum Beat Spectroscopy. The Journal of Physical Chemistry Letters 2015, 6, 35603564.

(9) Nibbering, E. T. J.; Dreyer, J.; Kühn, O.; Bredenbeck, J.; Hamm, P.; Elsaesser, T. In Analysis and Control of Ultrafast Photoinduced Reactions; Kühn, Oliver and Wöste, Ludger,, Ed.; Chemical Physics; Springer Berlin Heidelberg, 2007; Vol. 87; pp 619-687.

(10) Rury, A. S. Examining Resonant Inelastic Spontaneous Scattering of Classical LaguerreGauss Beams from Molecules. Physical Review A 2013, 87, 043408.

(11) Lucarini, V.; Saarinen, J. J.; Peiponen, K.-E.; Vartiainen, E. M. In Kramers-Kronig Relations in Optical Materials Research; Rhodes, W. T., Ed.; Springer Series in Optical Sciences; Springer-Verlag Berlin Heidelberg, 2005; Vol. 110.

(12) Stancik, A. L.; Brauns, E. B. A Simple Asymmetric Lineshape for Fitting Infrared Absorption Spectra. Vibrational Spectroscopy 2008, 47, 66-69. 